\title{
PFA and complemented subspaces of $\ell_{\infty} / c_{0}$
}

\begin{abstract}
ALAN DOW
Abstract: The Banach space $\ell_{\infty} / c_{0}$ is isomorphic to the linear space of continuous functions on $\mathbb{N}^{*}$ with the supremum norm, $C\left(\mathbb{N}^{*}\right)$. Similarly, the canonical representation of the $\ell_{\infty}$ sum of $\ell_{\infty} / c_{0}$ is the Banach space of continuous functions on the closure of any non-compact cozero subset of $\mathbb{N}^{*}$. It is important to determine if there is a continuous linear lifting of this Banach space to a complemented subset of $C\left(\mathbb{N}^{*}\right)$. We show that PFA implies there is no such lifting.
\end{abstract}

2010 Mathematics Subject Classification 54A25 (primary); 03E35 (secondary)

Keywords: Stone-Cech, PFA, Banach space

\section{Introduction}

Our paper is motivated by the question (Drewnowski and Roberts [3], Leonard and Whitfield [6]) of whether or not $C\left(\mathbb{N}^{*}\right)$ is primary. A Banach space $X$ is primary if whenever $X$ is written as the sum $A \oplus B$ of complemented subspaces, then one of $A, B$ is isomorphic to $X$. Negrepontis [8, Corollary 3.2] showed that $\mathrm{CH}$ implies that the closure $Y$ of a non-compact cozero subset of $\mathbb{N}^{*}$ is a retract of $\mathbb{N}^{*}$, and, therefore, there is a norm bounded linear lifting of the Banach space $C(Y)$ to a complemented subset of $C\left(\mathbb{N}^{*}\right)$. Later, Drewnowski and Roberts [3] established that the existence of such a lifting implied that $C\left(\mathbb{N}^{*}\right)$ is primary. It is already known to be consistent that there is no such lifting; an even stronger result was shown to hold in the Cohen model in Brech and Koszmider [1]. However there is still a good reason to investigate this question under the hypothesis of the proper forcing axiom. We still have no clear path to deciding if $C\left(\mathbb{N}^{*}\right)$ is primary in the Cohen model but Koszmider [9, p577] has identified a very compelling conjecture (as we choose to call it) that $C\left(\mathbb{N}^{*}\right)$ is not primary in certain forcing extensions of PFA. Establishing properties of $C\left(\mathbb{N}^{*}\right)$ in these extensions is very similar to working within PFA itself (see Veličković [14], Steprāns [12], and Dow and Shelah [2]). We present our work as progress towards confirming that conjecture. The paper Grzech [4] announced similar results and gave reference to a paper in preparation for details. But even now, a number of years later, the details of a proof have not appeared and there appear to be problems with the sketch described in [4, p306-307]; 
we say more on this in Remark 2.1 after establishing more notation. Our own proof takes quite a different approach. It is modeled on the methods developed in Farah [5] and Shelah and Steprāns [11].

\section{PFA implies no lifting}

Let $\left\{A_{n}: n \in \omega\right\}$ be a partition of $\mathbb{N}$ into infinite sets. Let $Y$ be the open subset $\bigcup_{n} A_{n}^{*}$ of $\mathbb{N}^{*}$. Consider the subspace $E=\left\{f \in C\left(\mathbb{N}^{*}\right): f[Y]=\{0\}\right\}$. It is well-known (see Pearl [9, p574]) that there is a continuous lifting for $C(\bar{Y})$ if and only if the subspace $E$ is complemented in the Banach space $\left(C\left(\mathbb{N}^{*}\right),\|\cdot\|_{\infty}\right)$. We take as our definition of $E$ being complemented that there is a projection $P$ from $C\left(\mathbb{N}^{*}\right)$ to $E$ (a bounded linear operator) satisfying that $P^{2}(f)=P(f) \in E$ for all $f \in C\left(\mathbb{N}^{*}\right)$. Of course the norm of $P$ is defined as the supremum of $\left\{\|P(f)\|_{\infty}:\|f\|_{\infty}=1\right\}$.

This then provides a complement to $E$ and an operator $T$ defined by $T(f)=f-P(f)$ for $f \in C\left(\mathbb{N}^{*}\right)$ onto that complement. Again, it follows that $T$ is bounded, linear, and satisfies that $T^{2}(f)=T(f)$. We may view $T$ as a lifting of the functions from $C(\bar{Y})$ into $C\left(\mathbb{N}^{*}\right)$ since it follows that $T(f) \uparrow \bar{Y}=f \uparrow \bar{Y}$ for all $f \in C\left(\mathbb{N}^{*}\right)$. More precisely, for any $h \in C(\bar{Y})$, define $H(h)$ to be $T(f)$ where $f$ is any $f \in C\left(\mathbb{N}^{*}\right)$ such that $h \subset f$. Then $H$ is a continuous linear embedding (in fact, lifting) of $C(\bar{Y})$ into $C\left(\mathbb{N}^{*}\right)$.

Theorem 1 (PFA) If $\left\{A_{n}: n \in \omega\right\}$ is a partition of $\mathbb{N}$ into infinite sets, then the subspace $E=\left\{f \in C\left(\mathbb{N}^{*}\right): f\left[\bigcup_{n} A_{n}^{*}\right]=\{0\}\right\}$ is not complemented. Equivalently, there is no operator $T$ as described above.

We assume PFA for the remainder and that $T$ is an operator as described in the paragraph immediately preceding the statement of the theorem. Following standard Stone-Cech compactification notation, the set of bounded (continuous) functions on $\mathbb{N}$ is denoted as $C^{*}(\mathbb{N})$. We fix any lifting of $T$ to all of $C^{*}(\mathbb{N})$ in the sense that for all bounded $f \in C(\mathbb{N}), T(f) \in C^{*}(\mathbb{N})$ is chosen so that $[T(f)]^{*}$ is equal to $T\left(f^{*}\right)$.

So, we note that, for all $f \in C^{*}(\mathbb{N}),(f-T(f))\left\lceil A_{n} \rightarrow 0\right.$ for all $n$. Additionally, for $f \in C^{*}\left(\mathbb{N}\right.$ ), we note that $\left\|f^{*}\right\|_{\infty}=0$ (ie $f^{*} \equiv 0$ ) is equivalent to $f$ converging to 0 on $\mathbb{N}$. We will say that two real-valued functions on $\mathbb{N}$ asymptotically agree if their difference converges to 0 . Also, when we refer to the norm of a member of $C^{*}(\mathbb{N})$ we mean the asymptotic norm or the norm of $f^{*}$.

The set $\left\{A_{n}: n \in \omega\right\}^{\perp}$ is the ideal of subsets of $\mathbb{N}$ which are almost disjoint from each $A_{n}$. Let $\mathcal{I}$ denote the larger (dense) ideal of sets that are almost disjoint from $A_{n}$ for all 
but finitely many $n$. As usual, $\mathcal{I}^{+}$is the collection of sets which are not in this ideal. Note that a set $a \subset \mathbb{N}$ is in $\mathcal{I}^{+}$if and only if the set $J_{a}=\left\{j:\left|a \cap A_{j}\right|=\omega\right\}$ is infinite. Unless mentioned otherwise, we will assume that $a \cap A_{n}$ is empty for $n \notin J_{a}$. Let $\mathcal{J} \subset \mathcal{I}^{+}$denote the collection of those $a \in \mathcal{I}^{+}$with the property that $J_{\mathbb{N} \backslash a}=\omega$. For any $a \in \mathcal{I}^{+}$, let $1_{a}$ denote the characteristic function. Therefore, for any $\rho \in C^{*}(\mathbb{N})$, $\rho \cdot 1_{a}$ is a function which is constantly 0 on $\mathbb{N} \backslash a$.

Remark 2.1 It is well-known that, in models of $\mathrm{CH}$, a continuous linear lifting $H$ of $C\left(\overline{\bigcup_{n} A_{n}^{*}}\right)$ into $C\left(\mathbb{N}^{*}\right)$ need not have the property that $H(f) \cdot H(g)=0$ whenever $f \cdot g=0$. This is similar to the fact that it is nearly immediate that if $H$ is a linear isomorphism between function spaces $C(X)$ and $C(Z)$, for $X, Z$ compact and $X$ zero-dimensional, and if $H$ satisfies that $H(f) \cdot H(g)=0$ whenever $f$ and $g$ are characteristic functions of disjoint clopen sets, then $X$ and $Z$ are homeomorphic. On the other hand, Miljutin[7] proved the surprising fact that $C\left(2^{\omega}\right)$ is linearly isomorphic to $C([0,1])$ (for example).

One quite incomplete step in the outline of the proof in Grzech [4] is connected to this aspect of linear isomorphisms. Conditions (2.5) and (2.6) on Page 307 of [4] seem to be essentially making this assumption about the isomorphism $H$ discussed. For example, it is very hard to see how to fulfill property (2.6) without having shown that if $\chi_{0} \cdot F=0$, then $H\left(\chi_{0}\right) \cdot H(F)=0$.

Comments on the proof: Many readers will know of Shelah's original method [10] for making an existing non-trivial automorphism of $\mathcal{P}(\mathbb{N}) / f$ in non-extendable in a generic extension. An almost disjoint family $\left\{a_{\alpha}: \alpha \in \omega_{1}\right\}$ of infinite subsets of $\mathbb{N}$ is constructed together with a family $\left\{b_{\alpha}: \alpha \in \omega_{1}\right\}$ of partitioners (ie $b_{\alpha} \subset a_{\alpha}$ ) in such a way that there is a ccc poset $\mathbb{P}_{\left\langle a_{\alpha}, b_{\alpha}: \alpha \in \omega_{1}\right\rangle}$ which forces the existence of a uniformizing partition $X$ satisfying that $X \cap a_{\alpha}={ }^{*} b_{\alpha}$ for each $\alpha \in \omega_{1}$ while preserving that there is no similar uniformizing $Y$ for the family $\left\{\varphi\left(a_{\alpha}\right), \varphi\left(b_{\alpha}\right): \alpha \in \omega_{1}\right\}$ (because it will contain a Hausdorff-Luzin type of gap). Clearly any possible value for $\varphi(X)$ must be such a uniformizing $Y$. The set-theoretic principle $\diamond$ is used to help ensure that the poset is ccc. Our method in this paper is based on this approach. We intend to similarly choose a sequence of sets $\left\{a_{\alpha}: \alpha \in \omega_{1}\right\} \subset \mathcal{J}$ and replace choosing $b_{\alpha}$ (or rather $1_{b_{\alpha}}$ ) by choosing some $f_{\alpha} \in C^{*}(\mathbb{N})$ with support contained in $a_{\alpha}$ (ie $f_{\alpha} \cdot 1_{\mathbb{N} \backslash a_{\alpha}}=0$ ) and again making these choices in such a way that we can force the existence of a uniformizing function $f_{\omega_{1}}$ in the sense that $f_{\omega_{1}} \cdot 1_{a_{\alpha}}$ asymptotically agrees with $f_{\alpha}$ for all $\alpha \in \omega_{1}$. However, the main new obstacle is that while $\varphi\left(b_{\alpha}\right)$ has no interaction with $\varphi\left(a_{\beta}\right)$ for $\beta \neq \alpha$, as remarked above, this is very much not the case with $T\left(f_{\alpha}\right) \cdot T\left(1_{a_{\beta}}\right)$. 
This makes it seemingly impossible to control for the possible existence of a function $g$ which might take the value for $T\left(f_{\omega_{1}}\right)$. That is, there is no expectation that $T\left(f_{\omega_{1}}\right) \cdot T\left(1_{a_{\beta}}\right)$ should have any sort of clear relationship to $T\left(f_{\beta}\right) \cdot T\left(1_{a_{\beta}}\right)$. To handle this we first prove (Lemma 2) the existence of " $T$-orthogonal pairs" $a, c$, subsets of $\mathbb{N}$, satisfying that $T\left(\rho \cdot 1_{c}\right) \cdot 1_{a}$ converges to 0 for all $\rho \in C^{*}(\mathbb{N})$. After proving the existence of such $T$-orthogonal pairs, we describe the construction of the poset $\mathbb{P}_{\left\langle f_{\alpha}, d_{\alpha}: \alpha \in \omega_{1}\right\rangle}$ (where for other technical reasons $\left\langle d_{\alpha}: \alpha \in \omega_{1}\right\rangle$ is a mod finite increasing sequence and the above mentioned $a_{\alpha}$ is contained in $d_{\alpha+1} \backslash d_{\alpha}$ ). While constructing this family, we also build in the construction of a suitable Hausdorff-Luzin type gap canonically coded by the family $\left\langle T\left(f_{\alpha+1}\right): \alpha \in \omega_{1}\right\rangle$ which will serve as the device for ensuring that no value for $T\left(f_{\omega_{1}}\right)$ will exist. The paper finishes with the necessary lemmas to show that the construction can be carried out.

Let $C_{1}$ be the set of functions from $\mathbb{N}$ into $\{-1,0,1\}$, and let $C_{1}^{+}$denote the set of functions from $\mathbb{N}$ into $\{0,1\}$. For any function $\rho \in C_{1}$, let $\rho^{+}, \rho^{-}$be the unique members of $C_{1}^{+}$such that $\rho=\rho^{+}-\rho^{-}$and $|\rho|=\rho^{+}+\rho^{-}$.

Lemma 2 Given $a, c \in \mathcal{I}^{+}$, there are $a_{1}, c_{1} \in \mathcal{I}^{+}$such that $a_{1} \subset a, J_{a_{1}}=J_{a}, c_{1} \subset c$, and for all $\rho \in C^{*}(\mathbb{N}),\left(T\left(\rho \cdot 1_{a_{1}}\right)\right) \cdot 1_{c_{1}}$ converges to 0 .

Proof We may assume that $a \cap c$ is empty. Since we are assuming that $T$ is a lifting, let us note that for all $\rho \in C_{1}$, there is a $B \in\left\{A_{n}: n \in \omega\right\}^{\perp}$ such that $T\left(\rho \cdot 1_{a}\right) \cdot 1_{\omega \backslash(a \cup B)}$ converges to 0 . In particular then we have that $T\left(\rho \cdot 1_{a}\right) \cdot 1_{c \backslash B}$ converges to 0 . This also implies that $T\left(\rho \cdot 1_{a}\right) \cdot 1_{c}$ is asymptotically equal to $T\left(\rho \cdot 1_{a}\right) \cdot 1_{c \backslash \bigcup_{j<n} A_{j}}$ for each $n \in \omega$.

Let $\mathcal{L}$ denote the set of pairs $\left(a_{1}, c_{1}\right)$ satisfying that $a_{1} \subset a, c_{1} \subset c, J_{a_{1}}=J_{a}$, and $c_{1} \in \mathcal{I}^{+}$. For each $\left(a_{1}, c_{1}\right) \in \mathcal{L}$, let the real number $L_{a_{1}, c_{1}}$ denote the least upper bound of the asymptotic norms of each member of the family $\left\{T\left(\rho \cdot 1_{a_{1}}\right) \cdot 1_{c_{1}}: \rho \in C_{1}\right\}$. Also let $L_{a_{1}, c_{1}}^{\downarrow}=\inf \left\{L_{a_{2}, c_{2}}:\left(a_{2}, c_{2}\right) \in \mathcal{L}\right.$ and $\left.a_{2} \subset a_{1}, c_{2} \subset c_{1}\right\}$.

Claim 1 There is a pair $\left(a_{1}, c_{1}\right) \in \mathcal{L}$ such that $L_{a_{1}, c_{1}}=L_{a_{1}, c_{1}}^{\downarrow}$.

Proof of Claim Let $\left(a_{0}, c_{0}\right)=(a, c)$ and recursively choose a pairwise descending sequence $\left\{\left(a_{n}, c_{n}\right): n \in \omega\right\} \subset \mathcal{L}$ so that $L_{a_{n+1}, c_{n+1}}<L_{a_{n}, c_{n}}^{\downarrow}+\frac{1}{2^{n}}$. Notice that for each $n$, we have that $L_{a_{n}, c_{n}}^{\downarrow} \leq L_{a_{n+1}, c_{n+1}}^{\downarrow} \leq L_{a_{n+1}, c_{n+1}} \leq L_{a_{n}, c_{n}}$. Choose any set $a_{\omega} \subset \bigcup_{j \in J_{a}} A_{j}$ so that $J_{a_{\omega}}=J_{a}$ and for each $j \in J_{a}, a_{\omega} \cap A_{j} \subset a_{j}$ and for each $n, a_{\omega} \cap A_{j} \subset^{*} a_{n}$. Notice that $a_{\omega} \backslash a_{n}$ is finite for all $n$. Choose a strictly increasing sequence $\left\{i_{n}: n \in \omega\right\}$ so that for each $n, c_{n} \cap A_{i_{n}}$ is infinite. Set $c_{\omega}=\bigcup_{n \in \omega} c_{n} \cap A_{i_{n}}$. We have that $\left(a_{\omega}, c_{\omega}\right) \in \mathcal{L}$, and that $c_{\omega} \backslash c_{n} \subset \bigcup_{i<i_{n}} A_{i}$ for all $n$. 
Let $\rho$ be any member of $C_{1}$ and let $n \in \omega$. We have that $\rho \cdot 1_{a_{\omega}}$ is mod finite equal to $\left(\rho \cdot 1_{a_{\omega}}\right) \cdot 1_{a_{n}}$. Therefore $T\left(\rho \cdot 1_{a_{\omega}}\right)$ is asymptotically equal to $T\left(\left(\rho \cdot 1_{a_{\omega}}\right) \cdot 1_{a_{n}}\right)$. Since the asymptotic norm of $T\left(\rho \cdot 1_{a_{n}}\right) \cdot 1_{c_{\omega}}$ is less than or equal to that of $T\left(\rho \cdot 1_{a_{n}}\right) \cdot 1_{c_{n}}$, we have that the asymptotic norm of $T\left(\rho \cdot 1_{a_{\omega}}\right) \cdot 1_{c_{\omega}}$ is bounded above by each $L_{a_{n}, c_{n}}$. By similar reasoning, it follows that $L_{a_{\omega}, c_{\omega}}^{\downarrow}$ is bounded below by $L_{a_{n}, c_{n}}^{\downarrow}$ for each $n$. This completes the proof of the claim.

Now that we have proven Claim 1, we may simply assume that $L=L_{a, c}$ is equal to $L_{a_{1}, c_{1}}$ for all $\left(a_{1}, c_{1}\right) \in \mathcal{L}$.

Claim 2 Suppose that $\left(a_{1}, c_{1}\right)$ and $\left(a_{2}, c_{2}\right)$ are in $\mathcal{L}$ and that $a_{1} \cap a_{2}$ is finite. Suppose also that $\rho_{1}, \rho_{2}$ are in $C_{1}$ and that for some $b \subset c_{1}$ and some $\epsilon>0$, the sequence $\left\{\left|T\left(\rho_{1} \cdot 1_{a_{1}}\right)(k)\right|: k \in b\right\}$ has no values below $L-\epsilon$. Then the asymptotic norm of the function $T\left(\rho_{2} \cdot 1_{a_{2}}\right) \cdot 1_{b}$ is at most $\epsilon$.

Proof of Claim Since $\left(a_{1} \cup a_{2}, c_{1}\right)$ is in $\mathcal{L}$ and $a_{1}$ and $a_{2}$ are disjoint, we have that each of $T\left(\rho_{1} \cdot 1_{a_{1}}+\rho_{2} \cdot 1_{a_{2}}\right) \cdot 1_{c_{1}}$ and $T\left(\rho_{1} \cdot 1_{a_{1}}-\rho_{2} \cdot 1_{a_{2}}\right) \cdot 1_{c_{1}}$ have norm at most $L$. We also have that each of $\left(T\left(\rho_{1} \cdot 1_{a_{1}}\right)+T\left(\rho_{2} \cdot 1_{a_{2}}\right)\right) \cdot 1_{b}$ and $\left(T\left(\rho_{1} \cdot 1_{a_{1}}\right)-T\left(\rho_{2} \cdot 1_{a_{2}}\right)\right) \cdot 1_{b}$ have norm at most $L$. The conclusion is then obvious.

The sets $C_{1}$ and $C_{1}^{+}$will be given the usual finite agreement topologies.

Claim 3 For each $\left(a_{1}, c_{1}\right) \in \mathcal{L}$ and each $\epsilon>0$, the set of $\rho \in C_{1}$ such that $T\left(\rho \cdot 1_{a_{1}}\right) \cdot 1_{c_{1}}$ has norm greater than $L-\epsilon$ is non-meager.

Proof of Claim Choose any $\epsilon>0$ and assume that $\left\{U_{n}: n \in \omega\right\}$ is a descending family of dense open subsets of $C_{1}$. There is a strictly increasing sequence $\left\{k_{n}\right.$ : $n \in \omega\} \subset \omega$ and functions $t_{n}:\left[k_{n}, k_{n+1}\right) \rightarrow\{0,1\}$ with the property that, for all $s \in\{0,1\}^{k_{n}}$, the basic clopen set $\left[s \cup t_{n}\right]$ is contained in $U_{n}$. We additionally require that $\left[k_{n}, k_{n+1}\right) \cap A_{j}$ is not empty for each $j \in J_{a} \cap n$. Let $a_{2}=\bigcup_{n}\left[k_{2 n}, k_{2 n+1}\right)$ and note that $a_{3}=a \backslash a_{2}$ satisfies that $J_{a_{3}}=J_{a}$.

Let $\rho_{2} \in C_{1}$ be any function such that $t_{2 n} \subset \rho_{2}$ for all $n$. Observe that for all $\psi \in C_{1}$, the function $\rho_{2} \cdot 1_{a_{2}}+\psi \cdot 1_{a_{3}}$ is in $U_{n}$ for each $n$. Choose $B \in\left\{A_{n}: n \in \omega\right\}^{\perp}$ so that $T\left(\rho_{2} \cdot 1_{a_{2}}\right) \cdot 1_{c_{1} \backslash B}$ converges to 0 . Choose $\psi \in C_{1}$ so that $T\left(\psi \cdot 1_{a_{3}}\right) \cdot 1_{c \backslash B}$ has norm greater than $L-\epsilon$. Finish the proof of the claim by observing that $T\left(\rho_{2} \cdot 1_{a_{2}}+\psi \cdot 1_{a_{3}}\right) \cdot 1_{c_{1} \backslash B}$ is asymptotically equal to $T\left(\psi \cdot 1_{a_{3}}\right) \cdot 1_{c \backslash B}$ and so has norm greater than $L-\epsilon$. 
Next we want to separate the contributions of $\rho^{+}$and $\rho^{-}$to the norm of $T\left(\rho \cdot 1_{a_{1}}\right) \cdot 1_{c_{1}}$. Consider any $\rho \in C_{1}$ and $\left(a_{1}, c_{1}\right) \in \mathcal{L}$ and let $L_{\rho}$ denote the norm of $T\left(\rho \cdot 1_{a_{1}}\right) \cdot 1_{c_{1}}$. Let $\mathcal{B}^{+}\left(\rho, a_{1}, c_{1}\right)$ denote the collection of infinite sets (if any) $b \subset c_{1}$ such that $T\left(\rho \cdot 1_{a_{1}}\right)\lceil b$ converges to $L_{\rho}$. Similarly let $\mathcal{B}^{-}\left(\rho, a_{1}, c_{1}\right)$ denote the collection of infinite sets $b \subset c_{1}$ such that $T\left(\rho \cdot 1_{a_{1}}\right) \uparrow b$ converges to $-L_{\rho}$. We will identify four types of possible behavior. When $\mathcal{B}^{+}\left(\rho, a_{1}, c_{1}\right)$ is non-empty we will identify type 1 and type 2 . The case when $\mathcal{B}^{+}\left(\rho, a_{1}, c_{1}\right)$ is empty will be categorized as type 3 or type 4 . It will be completely symmetric in that if $\rho$ is type 3 or type 4 , then $-\rho$ will be type 1 or type 2 respectively.

Let us focus on the case when $\mathcal{B}^{+}\left(\rho, a_{1}, c_{1}\right)$ is non-empty. We define $v\left(\rho, a_{1}, c_{1}\right)$ connected to $T\left(\rho^{+} \cdot 1_{a_{1}}\right)$. Define $v\left(\rho, a_{1}, c_{1}\right)$ to be the supremum of the norms of the family $\left\{T\left(\rho^{+} \cdot 1_{a_{1}}\right) \cdot 1_{b}: b \in \mathcal{B}^{+}\left(\rho, a_{1}, c_{1}\right)\right\}$. Similarly define $w\left(\rho, a_{1}, c_{1}\right)$ to be the supremum of the norms of the family $\left\{T\left(\rho^{-} \cdot 1_{a_{1}}\right) \cdot 1_{b}: b \in \mathcal{B}^{+}\left(\rho, a_{1}, c_{1}\right)\right\}$. Notice that $L_{\rho} \leq v\left(\rho, a_{1}, c_{1}\right)+w\left(\rho, a_{1}, c_{1}\right)$, and so $\max \left(v\left(\rho, a_{1}, c_{1}\right), w\left(\rho, a_{1}, c_{1}\right)\right) \geq \frac{L_{\rho}}{2}$. We will categorize $\rho$ as type 1 for $\left(a_{1}, c_{1}\right)$, when $v\left(\rho, a_{1}, c_{1}\right) \geq \frac{L_{\rho}}{2}$.

Clearly, for each $\left(a_{1}, c_{1}\right) \in \mathcal{L}$ and each $\epsilon>0$, there is a non-meager set of $\rho$ with $L_{\rho}>L-\epsilon$ of one of the four types for $\left(a_{1}, c_{1}\right)$. Let $\mathcal{L}_{i}$ denote the set of $\left(a_{1}, c_{1}\right) \in \mathcal{L}$ for which, for each $\epsilon>0$, there is a non-meager set of $\rho$ with $L_{\rho}>L-\epsilon$ which is type $i$ for $\left(a_{1}, c_{1}\right)$. By redefining $(a, c)$ to be some member of $\mathcal{L}_{i}$, we may assume that for each $\left(a_{1}, c_{1}\right) \in \mathcal{L}$, there is an $\left(a_{2}, c_{2}\right) \in \mathcal{L}_{i}$ with $a_{2} \subset a_{1}$ and $c_{2} \subset c_{1}$. For the remainder of the proof we assume, by symmetry, that this is true of $\mathcal{L}_{1}$.

This leads to the next claim, and the conclusion that $\mathcal{L}_{1}=\mathcal{L}$.

Claim 4 For each $\left(a_{1}, c_{1}\right) \in \mathcal{L}_{1}$ and each $\epsilon>0$, there is a non-meager set of $\rho \in C_{1}$ such that there are infinite disjoint $b, d$ contained in $c_{1}$ so that

(1) the set $T\left(\rho \cdot 1_{a_{1}}\right)[b]$ only has values greater than $L-\epsilon$,

(2) the $T\left(\rho^{+} \cdot 1_{a_{1}}\right)[b]$ only has values greater than $\frac{L}{2}-\epsilon$,

(3) the set $T\left(-\rho \cdot 1_{a_{1}}\right)[d]$ only has values greater than $L-\epsilon$,

(4) the set $T\left(\rho^{-} \cdot 1_{a_{1}}\right)[d]$ only has values greater than $\frac{L}{2}-\epsilon$.

Proof of Claim Choose any $\epsilon>0$ and assume that $\left\{U_{n}: n \in \omega\right\}$ is a descending family of dense open subsets of $C_{1}$. Choose a strictly increasing sequence $\left\{k_{n}: n \in\right.$ $\omega\} \subset \omega$ and functions $t_{n}:\left[k_{n}, k_{n+1}\right) \rightarrow\{-1,0,1\}$ so that, for all $s \in\{-1,0,1\}^{k_{n}}$, the basic clopen set $\left[s \cup t_{n}\right]$ is contained in $U_{n}$. We again require that $\left[k_{n}, k_{n+1}\right) \cap A_{j} \cap a_{1}$ is not empty for each $j \in J_{a} \cap n$. Let $a_{2}=\bigcup_{n}\left[k_{2 n}, k_{2 n+1}\right)$ and choose disjoint $a_{3}, a_{4} \subset a_{1} \backslash a_{2}$ so that $J_{a_{3}}=J_{a_{4}}=J_{a}$. 
Let $\rho_{2} \in C_{1}$ be any function such that $t_{2 n} \subset \rho_{2}$ for all $n$. Observe that for all $\psi \in C_{1}$, the function $\rho_{2} \cdot 1_{a_{2}}+\psi \cdot 1_{a_{3} \cup a_{4}}$ is in $U_{n}$ for each $n$. Choose $B_{0} \in\left\{A_{n}: n \in \omega\right\}^{\perp}$ so that each of $T\left(\rho_{2} \cdot 1_{a_{2}} \cdot 1_{a_{1}}\right), T\left(\rho_{2}^{+} \cdot 1_{a_{2}} \cdot 1_{a_{1}}\right)$, and $T\left(\rho_{2}^{-} \cdot 1_{a_{2}} \cdot 1_{a_{1}}\right)$ converges to 0 on the set $c_{1} \backslash B_{0}$. By shrinking $a_{3}$ we may suppose that there is some $c_{3} \subset c_{1} \backslash B_{0}$ so that $\left(a_{3}, c_{3}\right) \in \mathcal{L}_{1}$. Therefore we can choose $\psi_{3} \in C_{1}$ and some $b \in \mathcal{B}^{+}\left(\psi_{3}, a_{3}, c_{3}\right)$ so that the function $T\left(\psi_{3}^{+} \cdot 1_{a_{3}}\right)$ only has values greater than $\frac{L}{2}-\frac{\epsilon}{4}$ on the set $b$.

Now choose $B_{1} \in\left\{A_{n}: n \in \omega\right\}^{\perp}$ containing $B_{0}$ so that each of $T\left(\rho_{2} \cdot 1_{a_{2}} \cdot 1_{a_{1}}+\psi_{3}\right.$. $\left.1_{a_{3}}\right) \cdot 1_{c_{1} \backslash B_{1}}, T\left(\left(\rho_{2} \cdot 1_{a_{2}} \cdot 1_{a_{1}}+\psi_{3} \cdot 1_{a_{3}}\right)^{+}\right) \cdot 1_{c_{1} \backslash B_{1}}$, and $T\left(\left(\rho_{2} \cdot 1_{a_{2}} \cdot 1_{a_{1}}+\psi_{3} \cdot 1_{a_{3}}\right)^{-}\right) \cdot 1_{c_{1} \backslash B_{1}}$ converges to 0 .

Similarly, by shrinking $a_{4}$, choose a function $\psi_{4} \in C_{1}$ and an infinite set $d \subset c_{1} \backslash B_{1}$ so that the image of $d$ by $T\left(\psi_{4} \cdot 1_{a_{4}}\right)$ has no values below $L-\epsilon$, and the image of $d$ by $T\left(\psi_{4}^{+} \cdot 1_{a_{4}}\right)$ has no values below $\frac{L}{2}-\epsilon$.

Now set $\rho=\rho_{2} \cdot 1_{a_{2}}+\psi_{3} \cdot 1_{a_{3}}-\psi_{4} \cdot 1_{a_{4}}$ which is a member of the dense $G_{\delta}$ set $\bigcap_{n} U_{n}$. By the choice of $B_{1}$ and the linearity of $T$, we have that $T\left(\rho \cdot 1_{a_{1}}\right)=$ $T\left(\rho_{2} \cdot 1_{a_{2}} \cdot 1_{a_{1}}+\psi_{3} \cdot 1_{a_{3}}-\psi_{4} \cdot 1_{a_{4}}\right)$ asymptotically agrees with $T\left(-\psi_{4} \cdot 1_{a_{4}}\right)$ on $d$. Similarly $T\left(\rho^{-} \cdot 1_{a_{1}}\right)$ asymptotically agrees with $T\left(\psi_{4} \cdot 1_{a_{4}}\right)$ on $d$. This proves that items (3) and (4) of the claim hold.

By Claim 2, we have that each of $T\left(\psi_{4} \cdot 1_{a_{4}}\right)$ and $T\left(\psi_{4}^{-} \cdot 1_{a_{4}}\right)$ converge to 0 along $b$. We also have that $T\left(\rho_{2} \cdot 1_{a_{2}} \cdot 1_{a_{1}}+\psi_{3} \cdot 1_{a_{3}}\right)$ asymptotically agrees with $T\left(\psi_{3} \cdot 1_{a_{3}}\right)$ along $b$; and $T\left(\rho_{2}^{+} \cdot 1_{a_{2}} \cdot 1_{a_{1}}+\psi_{3}^{+} \cdot 1_{a_{3}}\right)$ asymptotically agrees with $T\left(\psi_{3}^{+} \cdot 1_{a_{3}}\right)$ along $b$. Putting all this together we have that $T\left(\rho \cdot 1_{a_{1}}\right)$ asymptotically agrees with $T\left(\psi_{3} \cdot 1_{a_{3}}\right)$ along $b$, and $T\left(\rho^{+} \cdot 1_{a_{1}}\right)$ asymptotically agrees with $T\left(\psi_{3}^{+} \cdot 1_{a_{3}}\right)$ along $b$. This verifies items (1) and (2) of the claim.

Now we are ready to apply OCA arguments to continue the proof. For each $j \in J_{a}$, choose any injection $\psi_{j}$ from $2^{<\omega}$ into $a \cap A_{j}$. Also choose, for each $j \in J_{c}$, an injection $\sigma_{j}$ of $J_{c}$ into $A_{j} \cap c$. For each $r \in 2^{\omega}$, let $a_{r}$ denote the set $a_{r}=\left\{\psi_{j}(r\lceil\ell): j<\ell \in \omega\}\right.$.

Let $\mathcal{X}$ denote the collection of functions of the form $\rho=\rho \cdot 1_{a_{r}}$ for some $r \in 2^{\omega}$, and $\rho \in C_{1}$ so that Claim 4 holds for some pair $b, d \subset c$.

For $\rho \in \mathcal{X}$, let

$$
b_{\rho}=\left\{k \in c: T\left(\rho^{+}\right)(k)>.45 L \text { and } T(\rho)(k)>.9 L\right\}
$$

and

$$
d_{\rho}=\left\{k \in c: T\left(\rho^{-}\right)(k)>.45 L \text { and } T(\rho)(k)<-.9 L\right\} .
$$

We define an open relation $K_{0}$ on $[\mathcal{X}]^{2}$ as follows. A pair $\left(\rho_{r}, \rho_{s}\right) \in K_{0}$ providing 
(1) $r \neq s$ are members of $2^{\omega}$,

(2) $\rho_{r} \cdot 1_{a_{r}}=\rho_{r}$,

(3) $\rho_{s} \cdot 1_{a_{s}}=\rho_{s}$,

(4) $\rho_{r}$ and $\rho_{s}$ agree on $a_{r} \cap a_{s}$,

(5) there is a $k \in\left(b_{\rho_{r}} \cap d_{\rho_{s}}\right) \cup\left(b_{\rho_{s}} \cap d_{\rho_{r}}\right)$.

Assume that $\left\{\rho_{\alpha}: \alpha \in \omega_{1}\right\} \subset C_{1}$ and $\left\{r_{\alpha}: \alpha \in \omega_{1}\right\} \subset 2^{\omega}$ are such that $\left[\left\{\rho_{\alpha} \cdot 1_{a_{r_{\alpha}}}\right.\right.$ : $\left.\left.\alpha \in \omega_{1}\right\}\right]^{2}$ is contained in $K_{0}$. For each $\alpha$, let $a_{\alpha}=a_{r_{\alpha}}$ and assume, with no loss, that $\rho_{\alpha}=\rho_{\alpha} \cdot 1_{a_{\alpha}}$. For each $\alpha$, let $b_{\alpha}=b_{\rho_{\alpha}}$ and $d_{\alpha}=d_{\rho_{\alpha}}$ Because of (5) the family $\left\{\left(b_{\alpha}, d_{\alpha}\right): \alpha \in \omega_{1}\right\}$ forms a Luzin family and so there is no set $Y \subset \mathbb{N}$ and uncountable $\Gamma \subset \omega_{1}$ such that $Y \bmod$ finite separates the family $\left\{b_{\alpha}: \alpha \in \Gamma\right\}$ from the family $\left\{d_{\alpha}: \alpha \in \Gamma\right\}$.

We consider the functions $f^{+}, f^{-}$where, for each $k$,

$$
f^{+}(k)=\max \left\{\rho_{\alpha}^{+}(k): \alpha \in \omega_{1}\right\} \text { and } f^{-}(k)=\max \left\{\rho_{\alpha}^{-}(k): \alpha \in \omega_{1}\right\} .
$$

Also let $f=f^{+}-f^{-}$. Notice that $f \cdot 1_{a}=f$ and, for each $\alpha \in \omega_{1}, f \cdot 1_{a_{\alpha}}=\rho_{\alpha}$.

Claim 5 The liminf of $T(f)$ on $b_{\alpha}$ is at least $.8 L$

Proof of Claim Assume that $b$ is any infinite subset of $b_{\alpha}$ and assume that $T(f) \uparrow b$ converges to some $L_{b}$. By thinning $b$ we may also assume that each of $T\left(\rho_{\alpha}\right)\lceil b$ and $T\left(f \cdot 1_{a \backslash a_{\alpha}}\right)\left\lceil b\right.$ also converge. We know that $T\left(\rho_{\alpha}\right) \uparrow b$, converges to some value greater than or equal to $.9 L$. By Claim $2, T\left(f \cdot 1_{a \backslash a_{\alpha}}\right)\lceil b$ must converge to values with absolute value less than or equal to .1L.

Similarly, we have

Claim 6 The limsup of $T(f)$ on $d_{\alpha}$ is at most $-.8 L$.

Now that we have that $Y=T(f)^{-1}(0, \infty)$ will mod finite separate the entire family $\left\{b_{\alpha}: \alpha \in \omega_{1}\right\}$ from $\left\{d_{\alpha}: \alpha \in \omega_{1}\right\}$, there is evidently no such uncountable $K_{0}$ homogeneous set.

Therefore, by OCA, we deduce there is a countable family $\left\{\mathcal{Y}_{n}: n \in \omega\right\}$ which covers $\mathcal{X}$ with the property that $\left[\mathcal{Y}_{n}\right]^{2} \cap K_{0}$ is empty for all $n$. For each $n$, there is a countable $Y_{n}$ which is a dense subset of $\mathcal{Y}_{n}$ in the suitable metric topology inherited from $\mathcal{X}$.

Choose any selective ultrafilter $\mathcal{U}$ on $\omega$ such that $J_{c} \in \mathcal{U}$. For each $U \in \mathcal{U}$, let $\sigma[U]$ denote the set $\left\{\sigma_{j}(k): j, k \in U \cap J_{c}\right.$ and $\left.|U \cap k|>j\right\}$. The family $\{\sigma[U]: U \in \mathcal{U}\}$ is a 
base for an ultrafilter on $\mathbb{N}$. It is the $\mathcal{U}$-limit of the sequence $\left\{\sigma_{j}(\mathcal{U}): j \in J_{c}\right\}$. To see this, assume that $W \subset \mathbb{N}$ is such that $U_{W}=\left\{j \in J_{c}: \sigma_{j}^{-1}\left(W \cap A_{j}\right)=U_{j} \in \mathcal{U}\right\} \in \mathcal{U}$. Since $\mathcal{U}$ is selective, there is a $U \in \mathcal{U}$ such that $U \subset U_{W}$ and, for each $j \in U$, $U \backslash \bigcap_{\ell<j} U_{\ell}$ has cardinality less than $j$. It follows that $\sigma[U] \subset W$.

Fix any countable elementary submodel with each of the above objects as elements. For $\eta \in \mathcal{X}$, let $r_{\eta}$ denote the member of $2^{\omega}$ such that $\eta \cdot 1_{a_{r_{\eta}}}=\eta$. We will choose an $r \in 2^{\omega}$ and then recursively define a $\rho \in C_{1}$ with $\rho \cdot 1_{a_{r}}=\rho$.

Let us consider any $s \in 2^{<\omega}$ and let $a^{s}=\left\{\psi_{j}(s \mid m): j<m<|s|\right\}$ which is the maximal common initial segment of $a_{r}$ for all $s \subset r \in 2^{\omega}$. Also fix any $\rho_{s}: a^{s} \rightarrow\{-1,0,1\}$. For any $n \in \omega$ and $s \subset t \in 2^{<\omega}$, define $\rho_{s, t} \supset \rho_{s}$ to be the function with domain $a^{t}$ which has value 0 on $a^{t} \backslash a^{s}$. We will consider the two sets

$$
W\left(\rho_{s}, n, t, 0\right)=\left\{k \in c:\left(\exists \eta \in \mathcal{Y}_{n}\right) \rho_{s, t} \subset \eta, t \subset r_{\eta} \text { and } T(\eta)(k)>.9 L\right\}
$$

and

$$
W\left(\rho_{s}, n, t, 1\right)=\left\{k \in c:\left(\exists \eta \in \mathcal{Y}_{n}\right) \rho_{s, t} \subset \eta, t \subset r_{\eta} \text { and } T(\eta)(k)<-.9 L\right\} .
$$

There is a sequence $\left\{U_{m}: m \in \omega\right\} \subset \mathcal{U}$ such that for each such $s, \rho_{s}, n, t$, there is an $m$ such that $\sigma\left[U_{m}\right]$ is either contained in, or disjoint from, $W\left(\rho_{s}, n, t, 0\right) \cap W\left(\rho_{s}, n, t, 1\right)$. Fix any $U \in \mathcal{U}$ which is mod finite contained in each $U_{m}$.

Choose any $r \in 2^{\omega}$ with the property that it does not contain any infinite chain of the form $E_{n, s, \rho_{s}}=\left\{t \in 2^{<\omega}: s \subset t\right.$, and $\left.W\left(\rho_{s}, n, t, 0\right) \cap W\left(\rho_{s}, n, t, 1\right) \in \mathcal{U}\right\}$ where $s \in\{r \mid \ell: \ell \in \omega\}, n \in \omega$, and $\rho_{s}: a^{s} \rightarrow\{-1,0,1\}$. In other words, if such an $E_{n, s, \rho_{s}}$ is contained in $r$, then it is finite. Since there are only countably many such chains, there is such an $r$.

Consider the forcing $\mathbb{P}_{r}$ consisting of finite approximations $\rho_{s}: a^{s} \rightarrow\{-1,0,1\}$ to a generic function $\rho: a_{r} \rightarrow\{-1,0,1\}$. Since $\left(a_{r}, \sigma[U]\right) \in \mathcal{L}_{1}$, whenever $\mathcal{D}$ is a countable family of dense subsets of $\mathbb{P}_{r}$, there will be a non-meager set of $\mathcal{D}$-generic $\rho$ that will satisfy that, not only is $\rho \in \mathcal{X}$, but also that $b_{\rho}$ and $d_{\rho}$ each hit $\sigma[U]$ in an infinite set.

Now for each integer $n$, define

$$
D_{n}=\left\{\rho_{s, t} \in \mathbb{P}_{r}: \text { either } t \notin E_{n, s, \rho_{s}} \text { or }\left(\exists \bar{t} \in E_{n, s, \rho_{s}}\right)(s \subset \bar{t} \perp t)\right\} .
$$

Fix any $\rho_{s} \in \mathbb{P}_{r}$. If $E_{n, s, \rho_{s}}$ is a chain, there is an extension $s \subset t \subset r$ such that $t \notin E_{n, s, \rho_{s}}$. Therefore $\rho_{s, t} \in D_{n}$. Otherwise, there is an extension $\bar{t} \supset s$ such that $\bar{t} \not \subset r$ and $\bar{t} \in E_{n, s, \rho_{s}}$. Choose any $s \subset t \subset r$ such that $t \perp \bar{t}$. Then we have that $\rho_{s, t} \in D_{n}$. This shows that $D_{n}$ is dense. 
Now we assume that $\rho$ is $\left\{D_{n}: n \in \omega\right\}$-generic over $\mathbb{P}_{r}$ and that $\rho \in \mathcal{X}$ and that each of $b_{\rho}$ and $d_{\rho}$ meet $\sigma[U]$ in an infinite set. Notice also that $b_{\rho}$ and $d_{\rho}$ necessarily meet each $c \cap A_{j}$ in a finite set. Therefore, $b_{\rho} \cap \sigma[U]$ and $d_{\rho} \cap \sigma[U]$ are mod finite contained in $\sigma\left[U_{m}\right]$ for each $m$. Consider any $n$ and assume that $\rho \in \mathcal{Y}_{n}$. By the density of $D_{n}$, there is an $s \subset t \subset r$ such that $\rho_{s, t} \subset \rho$ and $\rho_{s, t} \in D_{n}$. Choose the $\bar{t} \perp t$ so that $\rho_{s, \bar{t}} \in E_{n, s, \rho_{s}}$. Since there is an $m$ such that $\sigma\left[U_{m}\right] \subset W\left(\rho_{s}, n, \bar{t}, 0\right)$, there is a $k \in d_{\rho} \cap W\left(\rho_{s}, n, \bar{t}, 0\right)$. Choose $\eta \in \mathcal{Y}_{n}$ so that $\rho_{s, \bar{t}} \subset \eta, t \subset r_{\eta}$, and $T(\eta)(k)>.9 L$. We have now produced $\rho, \eta \in \mathcal{Y}_{n}$ such that $\{\rho, \eta\} \in K_{0}$.

This completes the proof of the lemma.

Let us say that a set $a$ is $T$-orthogonal to a set $c$ if for all $\rho \in C_{1}, T\left(\rho \cdot 1_{c}\right) \cdot 1_{a}$ converges to 0 . So far as we know, this is not a symmetric relation. Although it does follow from Lemma 2 that there are mutually $T$-orthogonal pairs, we do not know if there is such a choice with $c \in \mathcal{J}$ (as we will need), and so we are satisfied with the asymmetry.

Following a standard method of producing a proper poset for the application of PFA we pass to the $\mathrm{CH}$ extension obtained by forcing with $\omega_{2}^{<\omega_{1}}$. For any $h \in C_{1}$ and $d \in \mathcal{I}^{+}$, we define the poset $P_{h, d}$ to be the set of partial functions $p$ from $\mathbb{N}$ into $\{-1,0,1\}$ such that $\operatorname{dom}(p) \subset^{*} d$ and $p \subset^{*} h$ (in the sense of only finitely many disagreements). For any $\alpha \leq \omega_{1}$ and sequence $\left\langle f_{\beta}, d_{\beta}: \beta<\alpha\right\rangle$ of such $f_{\beta} \in C_{1}$ and $d_{\beta} \in \mathcal{I}^{+}$, satisfying that for $\beta<\gamma<\alpha d_{\beta} \subset^{*} d_{\gamma}$ and $f_{\gamma} \cdot 1_{d_{\beta}}={ }^{*} f_{\beta} \cdot 1_{d_{\beta}}$, the poset $P_{\left\langle f_{\beta}, d_{\beta}: \beta<\alpha\right\rangle}$ is defined to be $\bigcup_{\beta<\alpha} P_{f_{\beta}, d_{\beta}}$ We can fix a $\diamond$-sequence $\left\{S_{\alpha}: \alpha \in \omega_{1}\right\} \subset\left[\omega_{1}\right]^{\leq \omega}$ and fix an enumeration $\left\{H_{\alpha}: \alpha \in \omega_{1}\right\}$ of $H\left(\omega_{1}\right)$ (the hereditarily countable sets).

Now we define a sequence $\left\{d_{\beta}, f_{\beta}, \rho_{\beta}, a_{\beta}, \mathcal{D}_{\beta}: \beta \in \omega_{1}\right\}$ subject to the following inductive assumptions on $\alpha$ : for $\beta<\gamma<\alpha$,

(1) $d_{\gamma} \in \mathcal{J}$

(2) $a_{\beta} \subset d_{\gamma}, a_{\beta} \in \mathcal{I}^{+}$, and $d_{\beta} \cup a_{\beta} \subset d_{\beta+1}$,

(3) $f_{\beta}, f_{\gamma} \in C_{1}$ and $f_{\beta}=f_{\beta} \cdot 1_{d_{\beta}}={ }^{*} f_{\gamma} \cdot 1_{d_{\beta}}$,

(4) $f_{\gamma} \cdot 1_{a_{\beta}}=\rho_{\beta}$,

(5) for all $\rho \in C_{1}, T\left(\rho \cdot 1_{\mathbb{N} \backslash d_{\gamma}}\right) \cdot 1_{a_{\beta}}$ converges to 0 ,

(6) $\mathcal{D}_{\beta}$ is a countable family of predense subsets of $P_{f_{\beta}, d_{\beta}}$,

(7) $\mathcal{D}_{\beta} \subset \mathcal{D}_{\gamma}$,

(8) if $D_{\gamma}=\left\{H_{\xi}: \xi \in S_{\gamma}\right\}$ is a predense subset of $P_{\left\langle f_{\beta}, d_{\beta}: \beta<\gamma\right\rangle}$, then $D_{\gamma} \in \mathcal{D}_{\gamma}$. 
This construction using $\diamond$ as in condition (8) will ensure that the poset $P_{\omega_{1}}=$ $P_{\left\langle f_{\beta}, d_{\beta}: \beta<\omega_{1}\right\rangle}$ is ccc. This is from Shelah's oracle chain condition method of Shelah [10, $\S \mathrm{IV}]$. We also work with a listing, $\left\{\dot{Y}_{\beta}: \beta<\omega_{1}\right\}$, of all nice $P_{\omega_{1}}$-names of subsets of $\mathbb{N}$ such that $\dot{Y}_{\beta}$ is a $P_{f_{\gamma}, d_{\gamma}}$-name (for any $\beta<\gamma$ ). And we add the inductive condition

(9) for $\beta<\gamma, P_{\left\langle f_{\xi}, d_{\xi}: \xi<\gamma\right\rangle}$ forces that $\dot{Y}_{\beta}$ does not mod finite separate $b_{\gamma}$ from $e_{\gamma}$, where $b_{\gamma}=\left\{k \in a_{\gamma}: T\left(f_{\gamma+1}\right)(k)>\frac{2}{3}\right\}$ and $e_{\gamma}=\left\{k \in a_{\gamma}: T\left(f_{\gamma+1}\right)(k)<\frac{1}{3}\right\}$.

After constructing $a_{\gamma}$ and $\rho_{\gamma}$, we are able to preserve the property in item (9) by adding a specific countable family of dense sets to $\mathcal{D}_{\gamma+1}$.

The construction of this sequence will be explained in a series of lemmas. However before doing so, we indicate how this will prove the main theorem. After forcing with $P_{\omega_{1}}$, we have that the family $\left\{b_{\gamma}, e_{\gamma}: \gamma \in \omega_{1}\right\}$ can not be $\sigma$-separated. This implies (Todorčević [13, Theorem 2] and Shelah and Steprāns [11, Lemma 2]) there is a proper poset $Q$ which introduces an uncountable $\Gamma \subset \omega_{1}$ so that the family $\left\{b_{\gamma}, e_{\gamma}: \gamma \in \Gamma\right\}$ is a Luzin family (it is unsplit in any proper forcing extension). Now, we meet $\omega_{1}$ many dense subsets of $\omega_{2}^{<\omega_{1}} * P_{\omega_{1}} * Q$ in order to decide on the generic function $f=f_{\omega_{1}}$ added by $P_{\omega_{1}}$, and the Luzin gap $\left\{b_{\gamma}, e_{\gamma}: \gamma \in \Gamma\right\}$ as well as the basic properties of the family as detailed in items (1) - (6). Notice that (by the inclusion of $\omega_{1}$ many dense subsets of $\left.P_{\omega_{1}}\right) f \cdot 1_{d_{\gamma}}$ is almost equal to $f_{\gamma}$. It follows then that $T(f)$ can not exist. This is because $Y=T(f)^{-1}\left(\left(\frac{1}{2}, \infty\right)\right)$ is required to split the Luzin gap. To see this we have to show that $T(f) \cdot 1_{b_{\gamma}}$ has liminf greater than $\frac{1}{2}$, while $T(f) \cdot 1_{e_{\gamma}}$ has limsup less than $\frac{1}{2}$. We consider $T(f) \cdot 1_{a_{\gamma}}$ as asymptotically equal to $T\left(f \cdot 1_{d_{\gamma+1}}\right) \cdot 1_{a_{\gamma}}+T\left(f \cdot 1_{\mathbb{N} \backslash d_{\gamma+1}}\right) \cdot 1_{a_{\gamma}}$. Items (3) and (5) ensure that this is asymptotically equal to $T\left(f_{\gamma+1}\right) \cdot 1_{a_{\gamma}}$. Therefore, $Y \cap a_{\gamma}$ does separate $b_{\gamma}$ and $c_{\gamma}$.

We construct, by induction on $\alpha \in \omega_{1}$, the sequences

$$
\left\langle f_{\beta}, d_{\beta}, \mathcal{D}_{\beta}: \beta<\alpha\right\rangle \cup\left\langle\rho_{\beta}, a_{\beta}: \beta+1<\alpha\right\rangle
$$

as per inductive items (1)-(9) above. We can start very simply with $d_{0}=\emptyset, f_{0}$ the constant 0 function, and $\mathcal{D}_{0}=\{\emptyset\}$.

If $\alpha$ is a limit ordinal, then the choices of $f_{\alpha}, d_{\alpha}$ and $\mathcal{D}_{\alpha}$ are handled at the end in Lemma 6 . Therefore, we can proceed by assuming that we have constructed the family

$$
\left\langle f_{\beta}, d_{\beta}, \mathcal{D}_{\beta}: \beta \leq \alpha\right\rangle \cup\left\langle\rho_{\beta}, a_{\beta}: \beta+1 \leq \alpha\right\rangle .
$$

The choices for $f_{\alpha+1}, d_{\alpha+1}, \mathcal{D}_{\alpha+1}$ together with $a_{\alpha}, \rho_{\alpha}$ are established in Lemma 5. We will need preparatory lemmas leading up to it.

This next lemma is (essentially) statement $(* 1)$ of Shelah [10, IV $\S 5$, p134]. We sketch a proof for the reader's convenience. 
Lemma 3 Assume that $h \in C_{1}$ and $d \in \mathcal{J}$ are such that $h \cdot 1_{d}=h$ and assume that $c \in \mathcal{I}^{+}$is disjoint from $d$. If $\mathcal{E}$ is a countable family of predense subsets of $P_{h, d}$, then there is an $a \subset c$ such that $J_{a}=J_{a \backslash c}=J_{c}$ so that for all $\rho \in C_{1}$ each $E \in \mathcal{E}$ is a predense subset of the poset $P_{h+\rho \cdot 1_{a}, d \cup a}$.

Moreover, given $c$ and $a$ as above let $d_{1}=d \cup(c \backslash a)$. Then there is an $h_{1}$ such that $h_{1} \cdot 1_{d_{1}}=h_{1}, h_{1} \cdot 1_{d}=h \cdot 1_{d}$, and such that for all $\rho \in C_{1}$ with $\rho \cdot 1_{a}=\rho$, each $E \in \mathcal{E}$ is a predense subset of $P_{h_{1}+\rho, d_{1} \cup a}$.

Proof Let $\left\{p_{\ell}: \ell \in \omega\right\}$ enumerate all members finite functions from the poset $P_{h, d}$. Let $p_{\ell} \oplus h$ denote the function $p_{\ell} \cup h \uparrow\left(d \backslash \operatorname{dom}\left(p_{\ell}\right)\right)$. Let $\left\{E_{\ell}: \ell \in \omega\right\}$ be a descending sequence of dense subsets of $P_{h, d}$ so that the downward closure of each $E \in \mathcal{E}$ contains one of them. Recursively define an increasing sequence $\left\langle n_{k}: k \in \omega\right\rangle$ of integers as follows. Let $n_{0}=0$ and given $n_{k}$ ensure that $n_{k+1}$ is large enough so that $\operatorname{dom}\left(p_{\ell}\right) \subset n_{k+1}$ for all $\ell<n_{k}$ and that there is some $\ell_{k}<n_{k+1}$ so that $\operatorname{dom}\left(p_{\ell_{k}}\right)$ is contained in $\left[n_{k}, n_{k+1}\right) \backslash d$, and, for all $\ell$ such that $\operatorname{dom}\left(p_{\ell}\right)=n_{k}, p_{\ell_{k}} \cup\left(p_{\ell} \oplus h\right) \in E_{k}$. In addition, ensure that $c \cap A_{j} \cap\left[n_{k}, n_{k+1}\right)$ is not empty for each $j \in J_{c} \cap n_{k}$.

Let $a=c \cap \bigcup\left\{\left[n_{2 k}, n_{2 k+1}\right): k \in \omega\right\}$. Note that $J_{a}=J_{c \backslash a}=J_{c}$. Let $\rho$ be any member of $C_{1}$ and fix any $E \in \mathcal{E}$. We check that $E$ is predense in $P_{h+\rho \cdot 1_{a}, d \cup a}$. To do so we consider any $q \in P_{h+\rho, d \cup a}$. By extending $q$ we may assume that $\operatorname{dom}(q)$ contains $d \cup a$. Choose $k$ large enough so that the downward closure of $E$ in $P_{h, d}$ contains $E_{k}$, $\operatorname{dom}(q) \subset d \cup a \cup n_{2 k+1}$, and such that $q(j)=(h+\rho)(j)$ for all $n_{2 k+1}<j \in d \cup a$. There is an $\ell$ such that $q\left\lceil n_{2 k+1}\right.$ is contained in $p_{\ell}$ and $\operatorname{dom}\left(p_{\ell}\right)=n_{2 k+1}$. By construction $p_{\ell_{2 k+1}} \cup\left(p_{\ell} \oplus h\right)$ is in $E_{2 k}$. Since $p_{\ell_{2 k+1}} \cup\left(p_{\ell} \oplus h\right)$ is contained in $p_{\ell_{2 k+1}} \cup q$, we have that $q$ is compatible with a member of $E$.

Now assume that $d \cup c \in \mathcal{J}$ and choose $h_{1} \in C_{1}$ so that $h_{1} \cdot 1_{d}=h \cdot 1_{d}$ and so that $h\left\lceil c=\bigcup\left\{p_{\ell_{k}}\lceil c: k \in \omega\}\right.\right.$. Also ensure that $h_{1} \cdot 1_{\mathbb{N} \backslash d_{1}}$ is 0 . The same argument as above shows that each $E \in \mathcal{E}$ is predense in $P_{h_{1}, d_{1}}$ because $p_{k}\left\lceil c \subset h_{1}\right.$ for all $k$.

Having chosen $f_{\alpha}, d_{\alpha}$, we are ready to choose $a_{\alpha}$. First apply Lemma 2 to find $\tilde{a}_{\alpha} \in \mathcal{I}^{+}$ and disjoint $c_{\alpha} \subset \mathbb{N} \backslash d_{\alpha}$ so that $\tilde{a}_{\alpha}$ is $T$-orthogonal to $c_{\alpha}$ and so that $J_{c_{\alpha}}=\omega$. Next apply Lemma 3 (with $c=\tilde{a}_{\alpha}$ ) to choose any $a_{\alpha} \in \mathcal{I}^{+}$contained in $\tilde{a}_{\alpha}$ and $h_{\alpha, 0}$ so that $h_{\alpha, 0} \cdot 1_{d_{\alpha}}=f_{\alpha}, h_{\alpha, 0} \cdot 1_{a_{\alpha} \cup c_{\alpha}}=0$ such that we are free to choose any $\rho_{\alpha} \in C_{1}$ with $\rho_{\alpha}=\rho_{\alpha} \cdot 1_{\tilde{a}_{\alpha}}$ so as to preserve that each member of the family $\mathcal{D}_{\alpha}$ is predense in the poset $P_{h_{\alpha, 0}+\rho_{\alpha}, \mathbb{N} \backslash\left(a_{\alpha} \cup c_{\alpha}\right)}$. Set $d_{\alpha, 0}=\mathbb{N} \backslash\left(a_{\alpha} \cup c_{\alpha}\right)$.

With this reduction, we have now guaranteed that with this choice of $a_{\alpha}$ and $d_{\alpha+1}=$ $\mathbb{N} \backslash c_{\alpha}$, then for all $\gamma>\alpha$, so long as $f_{\gamma} \cdot 1_{d_{\alpha+1}}={ }^{*} f_{\alpha+1}=f_{\alpha+1} \cdot 1_{d_{\alpha+1}}$ (as in inductive 
condition (3)) is satisfied, then $T\left(f_{\gamma}\right) \cdot 1_{a_{\alpha}}$ will be asymptotically equal to $T\left(f_{\alpha+1}\right) \cdot 1_{a_{\alpha}}$. The reason is that $T\left(f_{\gamma}\right)-T\left(f_{\alpha+1}\right)$ will be asymptotically equal to $T\left(f_{\gamma} \cdot 1_{c_{\alpha}}\right)$, and $a_{\alpha}$ is $T$-orthogonal to $c_{\alpha}$.

The key property of the choice of $\rho_{\alpha}$ is the requirement on $\dot{Y}_{\beta}$ for each $\beta<\alpha$. This next lemma shows how to handle one such $\beta$, then we extend to all countably many in the subsequent lemma.

Lemma 4 Let $a, d$ be disjoint members of $\mathcal{I}^{+}$and let $h \in C_{1}$ be such that $h \cdot 1_{d}=h$. Further suppose that $\dot{Y}$ is a $P_{h, d}$-name for a subset of $\mathbb{N}$ and let $p_{0}$ be any member of $P_{h, d}$. Then there is a $\rho \in C_{1}$ such that, $p_{0} \subset \rho, \rho \cdot 1_{d \cup a}={ }^{*} \rho$, and such that $\rho \uparrow(d \cup a)$ forces, with respect to the poset $P_{h+\rho \cdot 1_{a}, d \cup a}$, that $\dot{Y}$ does not mod finite separate $a \cap T(\rho)^{-1}\left(\frac{2}{3}, \infty\right)$ and $a \cap T(\rho)^{-1}\left(-\infty, \frac{1}{3}\right)$.

Proof Assume that $\dot{Y}$ is such a name and that there is no such $\rho$. Fix any integer $L$, we will prove that $T$ has norm exceeding $L$. We may obviously assume that $a$ is disjoint from $\operatorname{dom}\left(p_{0}\right)$ and that $\operatorname{dom}\left(p_{0}\right) \supset d$. We may assume that $\dot{Y}$ is a simple name that is a subset of $\mathbb{N} \times P_{h, d}$ and, for a generic filter $G, \operatorname{val}_{G}(\dot{Y})=\{k:(\exists r \in G)(k, r) \in \dot{Y}\}$. Let $p_{0} \overline{0} \in C_{1}$ denote the extension of $p_{0}$ satisfying that $p_{0} \overline{0} \cdot 1_{\operatorname{dom}\left(p_{0}\right)}=p_{0} \overline{0}$. By the properties of $T$ we have that $T\left(p_{0}^{\frown} \overline{0}\right)$ converges to 0 on $a \cap A_{j}$ for each $j \in J_{a}$. By removing a finite set from each $a \cap A_{j}$, we may assume that $T\left(p_{0}^{-} \overline{0}\right)(k)$ has absolute value less than $\frac{1}{9}$ for all $k \in a$.

Fix, for each $j \in J_{a}$ an injection $\psi_{j}: 2^{<\omega} \rightarrow a \cap A_{j}$. Our plan is to choose $\rho \in C_{1}$ so that for all $j, x_{\rho, j}=\left\{s \in 2^{<\omega}: \rho\left(\psi_{j}(s)\right) \neq 0\right\}$ is a chain. Let $Q \subset P_{h, d}$ denote the set of those $p \in P_{h, d}$ with this same property, namely, that for all $j, x_{p, j}=\left\{s \in 2^{<\omega}\right.$ : $\left.p\left(\psi_{j}(s)\right) \neq 0\right\}$ is a (possibly empty) chain. Let $x_{p, j}^{+}=\left\{s \in x_{p, j}: p\left(\psi_{j}(s)\right)>\frac{7}{9}\right\}$ and $x_{p, j}^{-}=\left\{s \in x_{p, j}: p\left(\psi_{j}(s)\right)<\frac{2}{9}\right\}$. The ordering on $Q$, inherited from $P_{h, d}$, is that $r \leq_{Q} q$ providing $q \subseteq r$. We may consider $\dot{Y}$ (equivalently $\dot{Y} \cap(\mathbb{N} \times Q)$ ) as a $Q$-name. Fix an enumeration $\left\{q_{\ell}: \ell \in \omega\right\}$ of $\{q \in Q: \operatorname{dom}(q) \cap a=\emptyset\}$.

For any $j \in J_{a}$, say that an element $q \in Q$ is $j$-decisive if for all $q \subset r$ in $Q$, $r \Vdash_{Q} \psi_{j}(t) \in \dot{Y}$ for all $t \in x_{r, j}^{+} \backslash x_{q, j}$, and $r \Vdash_{Q} \psi_{j}(t) \notin \dot{Y}$ for all $t \in x_{r, j}^{-} \backslash x_{q, j}$.

Claim 7 For each $p_{0} \subseteq p \in Q$ and $j \in J_{a}$ there is a $p \subseteq q$ in $Q$ which is $j$-decisive.

If no such $q$ exists, then, we recursively choose an $\subset$-increasing sequence $\left\{r_{k}: k \in\right.$ $\omega\} \subset Q$ with $p=r_{0}$ and $\operatorname{dom}\left(r_{k} \backslash p\right) \subset a$ for all $k$. Also ensure that $\bigcup_{k} \operatorname{dom}\left(r_{k}\right)=a$. The inductive hypothesis is that for each $k$ and each $\ell<k$, if $q_{\ell} \cup r_{k} \in Q$, then either 
there is $\ell^{\prime}$ and a $t \in x_{r_{k+1}, j}^{+} \backslash x_{r_{k}, j}$ such that $q_{\ell^{\prime}} \cup r_{k+1} \in Q, q_{\ell^{\prime}} \cup r_{k+1}<q_{\ell} \cup r_{k}$, and $q_{\ell^{\prime}} \cup r_{k+1} \Vdash \psi_{j}(t) \notin \dot{Y}$, or a similar conclusion for some $t \in x_{r_{k+1}, j}^{-} \backslash x_{r_{k}, j}$.

Upon completion of this recursion, set $\rho=\bigcup_{k} r_{k}$. We check that $\rho$ is as required in the conclusion of the lemma. First of all, let us recall that $\rho$ and $T(\rho)$ are asymptotically equivalent on $a \cap A_{j}$. So there is an $k_{0}$ such that $\left|\rho\left(\psi_{j}(t)\right)-T(\rho)\left(\psi_{j}(t)\right)\right|<\frac{1}{9}$ for all $t \in \bigcup_{k} x_{r_{k}} \backslash x_{r_{k_{0}}}$.

Now let us assume that there is a $\bar{q} \in P_{\rho, d \cup a}$ extending $\rho\lceil(d \cup a)$, and an $m \in \omega$ such that $\bar{q}$ forces that $\dot{Y}$ contains $(a \backslash m) \cap A_{j} \cap T(\rho)^{-1}\left(\frac{2}{3}, \infty\right)$ and is disjoint from $(a \backslash m) \cap A_{j} \cap T(\rho)^{-1}\left(-\infty, \frac{1}{3}\right)$. By enlarging $k_{0}$, we can assume that $\psi_{j}(t)>m$ for all $t \in \bigcup_{k} x_{r_{k}} \backslash x_{r_{k_{0}}}$. Therefore we have that $\bar{q}$ forces that $\psi_{j}(t) \in \dot{Y}$ for all $t \in \bigcup_{k} x_{r_{k}}^{+} \backslash x_{r_{k_{0}}}$, and that $\psi_{j}(t) \notin \dot{Y}$ for all $t \in \bigcup_{k} x_{r_{k}}^{-} \backslash x_{r_{k_{0}}}$.

Set $q=\bar{q}\left\lceil(\mathbb{N} \backslash a)\right.$ and notice that $q \in Q$ and so there is an $\ell$ with $q_{\ell}=q$. Choose any $k>\ell, k_{0}$. By symmetry, since $q_{\ell}$ is not $j$-decisive, we may assume there is $t \in x_{r_{k+1}, j}^{+} \backslash x_{r_{k}, j}$ and an $\ell^{\prime}$ such that $q_{\ell^{\prime}} \cup r_{k+1} \Vdash_{Q} \psi_{j}(t) \notin \dot{Y}$. However, since $\operatorname{dom}\left(\rho \backslash r_{k+1}\right) \subset a$, we have that $q_{\ell^{\prime}} \cup \rho<\rho$ is in the poset $P_{\rho, d \cup a}=P_{h+\rho \cdot 1_{a}, d \cup a}$ and so, by the assumption on $\bar{q}$, forces that $\psi_{j}(t) \in \dot{Y}$. By our assumption on the name $\dot{Y}$, there is a condition $r \in P_{h, d}$ such that $\left(\psi_{j}(t), r\right) \in \dot{Y}$ and is such that $r \cup q_{\ell^{\prime}} \cup \rho$ is an extension of $q_{\ell^{\prime}} \cup \rho$. Of course then, $r \cup q_{\ell^{\prime}} \cup r_{k+1}$ forces that $\psi_{j}(t) \in \dot{Y}$ which contradicts that $q_{\ell^{\prime}} \cup r_{k+1} \Vdash_{Q} \psi_{j}(t) \notin \dot{Y}$.

Next we use the claim to show that $L$ is not a bound on the norm of $T$. The key idea is that being $j$-decisive is decidable and so we can build suitably long $\psi_{j}$-chains in $A_{j}$ and then branch away into $5 L$ many incomparable extensions that share an element $\psi_{j}(t)$ forced to be in $\dot{Y}$.

Claim 8 There is a doubly-indexed set $\left\{g_{i}^{k}: i \leq 5 L, k \in \omega\right\} \subset Q$ and an increasing sequence $\left\{j_{k}: k \in \omega\right\} \subset J_{a}$ such that, for each $k$ and $i \leq 5 L$

(1) $p_{0} \subset g_{i}^{k} \subset g_{i}^{k+1}$,

(2) $\operatorname{dom}\left(g_{i}^{k} \backslash p_{0}\right) \subset a$,

(3) for each $\ell<k$, there is an $\ell^{\prime}$ such that $q_{\ell} \subset q_{\ell^{\prime}}$ and $q_{\ell^{\prime}} \cup g_{i}^{k+1}$ is $j_{k}$-decisive,

(4) $g_{i}^{k+1} \uparrow\left(a \cap A_{j_{k}}\right) \subset g_{i+1}^{k+1} \uparrow\left(a \cap A_{j_{k}}\right)$ for $i<5 L$,

(5) there is a $t_{k} \in x_{g_{5 L}^{k+1}, j_{k}}^{+} \cap x_{g_{i}^{k+2}, j_{k}}^{+} \backslash x_{g_{i}^{k+1}, j_{k}}$,

(6) for all $j \in J_{a} \cap j_{k}$ and $i \neq \ell \leq 5 L, x_{g_{i}^{k+2}, j} \cup x_{g_{\ell}^{k+2}, j}$ is not a chain. 
Proof of Claim 8 We begin with $g_{i}^{0}=p_{0}$ for each $i \leq 5 L$ and $j_{-1}=0$. Assume that we have selected $j_{k-1}$ and $\left\{g_{i}^{k}: i \leq 5 L\right\}$ for some $k$. Set $\ell_{0}=k$. Choose any $j_{k}>j_{k-1}$ in $J_{a}$ so that $\operatorname{dom}\left(g_{i}^{k}\right) \cap a \cap A_{j_{k}}$ is empty for all $i \leq 5 L$. Choose any extension $\bar{g}_{0}^{k+1}$ of $g_{0}^{k} \cup\left(g_{5 L}^{k} \uparrow\left(a \cap A_{j_{k-1}}\right)\right)$ which is $j_{k}$-decisive. Suppose $i<5 L$ and we have chosen $\bar{g}_{i}^{k+1}$ and a value $\ell_{i+1}$ so that for each $\ell<\ell_{i}$ there is an $\ell^{\prime}<\ell_{i+1}$ such that $q_{\ell} \subset q_{\ell^{\prime}}$ and $q_{\ell^{\prime}} \cup \bar{g}_{i}^{k+1}$ is $j_{k}$-decisive. Choose $\bar{g}_{i+1}^{k+1}$ (in $\ell_{i+1}$ steps) to be any extension of $g_{i+1}^{k} \cup\left(g_{5 L}^{k} \uparrow\left(a \cap A_{j_{k-1}}\right)\right) \cup\left(\bar{g}_{i}^{k+1} \uparrow\left(a \cap A_{j_{k}}\right)\right)$ so that there is an $\ell_{i+2}$ such that for all $\ell<\ell_{i+1}$, there is an $\ell^{\prime}<\ell_{i+2}$ so that $q_{\ell} \subset q_{\ell^{\prime}}$ and $q_{\ell^{\prime}} \cup \bar{g}_{i+1}^{k+1}$ is $j_{k}$-decisive. When choosing $\bar{g}_{5 L}^{k+1}$ ensure also that there is $t_{k} \in x_{\bar{g}_{5 L}^{k+1}, j_{k}}^{+}$which is not in $x_{\bar{g}_{i}^{k+1}, j_{k}}$ for any $i<5 L$. Notice that this construction has ensured that $t_{k-1} \in x_{g_{i}^{k+1}, j_{k-1}}^{+}$for each $i \leq 5 L$. Finally, choose $g_{i}^{k+1}$ to be an extension of $\bar{g}_{i}^{k+1}$ so that $g_{i}^{k+1} \uparrow\left(a \cap A_{j_{k}}\right)=\bar{g}_{i}^{k+1} \uparrow\left(a \cap A_{j_{k}}\right)$ and in such a way that for all $j \in J_{a} \cap j_{k}$ and all distinct $\ell, i \leq 5 L, x_{g_{i}^{k+1}, j} \cup x_{g_{\ell}^{k+1}, j}$ is not a chain (this last step is a triviality).

Now, let us consider $g_{i}=\bigcup_{k \in \omega} g_{i}^{k}$ for each $i \leq 5 L$. But also, by the additional properties of $T$, we can choose $a_{1} \subset a$ so that for each $j \in J_{a}, a \cap A_{j} \backslash a_{1}$ is finite, and so that for all $i<\ell<5 L$, we have that $g_{i} \cdot g_{\ell} \cdot 1_{a_{1}}$ is constantly 0 . Then we have that $T\left(g_{i} \cdot 1_{a_{1}}\right)$ is asymptotically equal to $T\left(g_{i} \cdot 1_{a}\right)$ and $\Sigma_{i<5 L} g_{i} \cdot 1_{a_{1}}$ has norm at most 1 . Also, $T\left(\sum_{i<5 L} g_{i} \cdot 1_{a_{1}}\right)$ is asymptotically equal to $T\left(\sum_{i<5 L} g_{i} \cdot 1_{a}\right)$. By our assumption, we have that there is some $q_{\ell}$ which, for each $i \leq 5 L$ has decided on the $m$ and forces that for all $\sigma_{j}\left(t_{k}\right)>m$ which are in $\dot{Y}$, we must have that $T\left(g_{i} \cdot 1_{a}\right)\left(\sigma_{j}\left(t_{k}\right)\right)>\frac{1}{3}$.

But now if $q_{\bar{\ell}}$ is any extension of $q_{\ell}$, then for each $k>\bar{\ell}$, there is a further extension $q_{\ell^{\prime}}$ such that, for each $i<5 L, q_{\ell^{\prime}} \cup g_{i, j_{k}}^{k+1}$ is $j_{k}$-decisive. That is, $q_{\ell^{\prime}} \cup g_{i, j_{k}}^{k+1}$ forces that $\psi_{j_{k}}\left(t_{k}\right) \in \dot{Y}$. Therefore, it follows that $T\left(\sum_{i<5 L} g_{i} \cdot 1_{a_{1}}\right)\left(\psi_{j_{k}}\left(t_{k}\right)\right)$ is greater than $(5 L)\left(\frac{2}{9}\right)$ for infinitely many $k$. Which shows that the norm of $T$ is greater than $L$.

Lemma 5 Given $f_{\alpha}, d_{\alpha}$ and $\mathcal{D}_{\alpha}$ as in the inductive construction, there is an $a_{\alpha} \in \mathcal{I}^{+}$ which is disjoint from $d_{\alpha}$, a pair $f_{\alpha+1}, d_{\alpha+1}$, and a countable family $\mathcal{D}_{\alpha+1}$ such that for each $\beta<\alpha$

(1) $d_{\alpha} \cup a_{\alpha} \subset d_{\alpha+1}$,

(2) $d_{\alpha+1} \in \mathcal{J}$,

(3) $f_{\alpha+1} \cdot 1_{d_{\alpha}}=f_{\alpha}$, and $f_{\alpha+1} \cdot 1_{d_{\alpha+1}}=f_{\alpha+1}$,

(4) $a_{\alpha}$ is $T$-orthogonal to $c_{\alpha}=\mathbb{N} \backslash d_{\alpha+1}$,

(5) $\mathcal{D}_{\alpha} \subset \mathcal{D}_{\alpha+1}$ and each $D \in \mathcal{D}_{\alpha+1}$ is a predense subset of $P_{f_{\alpha+1}, d_{\alpha+1}}$,

(6) if $G$ is any $\mathcal{D}_{\alpha+1}$-generic filter on $P_{f_{\alpha+1}, d_{\alpha+1}}$, then $\operatorname{val}_{G}\left(\dot{Y}_{\beta}\right)$ does not mod finite separate $b_{\alpha}$ and $e_{\alpha}$. 
Proof As discussed before the previous lemma, there are $a_{\alpha}, c_{\alpha}$ and $h_{\alpha, 0} \in C_{1}$ and $d_{\alpha, 0}=\mathbb{N} \backslash\left(a_{\alpha} \cup c_{\alpha}\right)$ so that

(1) $d_{\alpha+1}=d_{\alpha, 0} \cup a_{\alpha} \in \mathcal{J}$,

(2) $h_{\alpha, 0} \cdot 1_{d_{\alpha}}=f_{\alpha}$, and $h_{\alpha, 0} \cdot 1_{a_{\alpha} \cup c_{\alpha}}=0$,

(3) for any $\rho_{\alpha} \in C_{1}$ with $\rho_{\alpha}=\rho_{\alpha} \cdot 1_{a_{\alpha}}$, each $D \in \mathcal{D}_{\alpha}$ is predense in $P_{h_{\alpha, 0}+\rho_{\alpha}, d_{\alpha, 0}}$,

(4) $a_{\alpha}$ is $T$-orthogonal to $c_{\alpha}$.

We will recursively choose disjoint infinite subsets $a_{\alpha, n}$ of $a_{\alpha}$ and functions $\rho_{\alpha, n}=$ $\rho_{\alpha} \cdot 1_{a_{\alpha, n}}$ so as to "handle" $\dot{Y}_{n}$. However, in doing so we have to take care when defining $a_{\alpha, n+1}$ to ensure that the full $\rho_{\alpha}$ will not change the fact that $\dot{Y}_{n}$ was appropriately handled by $\rho_{\alpha} \uparrow a_{\alpha, n}$. Let us again note that regardless of our choice of $\rho_{\alpha}$, each member of $\mathcal{D}_{\alpha}$ will be predense in $P_{h_{\alpha, 0}+\rho_{\alpha}, d_{\alpha+1}}$.

However, in order to make the first step general enough to handle all later steps, we may suppose we have some countable family $\mathcal{E}_{\alpha, 0}$ of predense subsets of $P_{h_{\alpha, 0}, \mathbb{N} \backslash\left(a_{\alpha} \cup c_{\alpha}\right)}$, that must be preserved. Fix any $p_{0} \in P_{h_{\alpha, 0}, \mathbb{N} \backslash\left(a_{\alpha} \cup c_{\alpha}\right)}$ and any $\dot{Y}_{\beta_{0}}$ with $\beta_{0}<\alpha$.

To begin, apply Lemma 2 to obtain disjoint subsets, $\tilde{a}_{\alpha, 0}$ and $c_{\alpha, 0}$, of $a_{\alpha}$ so that $\tilde{a}_{\alpha, 0}$ is $T$-orthogonal to $c_{\alpha, 0}$. These may be chosen so that each are in $\mathcal{I}^{+}$and are disjoint from $\operatorname{dom}\left(p_{0}\right)$. Apply Lemma 3 to choose $a_{\alpha, 0} \subset \tilde{a}_{\alpha, 0}$ and a function $h_{\alpha, 1} \in C_{1}$ so that $h_{\alpha, 1} \cdot 1_{d_{\alpha, 0}}=h_{\alpha, 0}, h_{\alpha, 1} \cdot 1_{a_{\alpha, 0} \cup c_{\alpha, 0}}=0$, and, for all $\rho \in C_{1}$ with $\rho \cdot 1_{a_{\alpha, 0} \cup c_{\alpha, 0}}=\rho$, we have that each member of $\mathcal{E}_{\alpha, 0}$ is predense in $P_{h_{\alpha, 1}+\rho, \mathbb{N} \backslash c_{\alpha}}$. Set $d_{\alpha, 1}=d_{\alpha, 0} \cup a_{\alpha} \backslash\left(a_{\alpha, 0} \cup c_{\alpha, 0}\right)$.

This gives us the poset $P_{h_{\alpha, 1}, d_{\alpha, 1}}$ and first we replace $p_{0}$ by the unique extension with domain $d_{\alpha, 1}$ which agrees with $h_{\alpha, 1}$ at all points not in $\operatorname{dom}\left(p_{0}\right)$. Then we apply Lemma 4 , and in this way we obtain $\rho_{\alpha, 0} \in C_{1}$ with $\rho_{\alpha, 0} \cdot 1_{a_{\alpha, 0}}=\rho_{\alpha, 0}$, so that $\left(p_{0}+\rho_{\alpha, 0}\right) \uparrow$ $\left(d_{\alpha, 1} \cup a_{\alpha, 0}\right)$ forces with respect to the poset $P_{h_{\alpha, 1}+\rho_{\alpha, 0}, d_{\alpha, 1} \cup a_{\alpha, 0}}$, that $\dot{Y}_{\beta_{0}}$ does not mod finite split $a_{\alpha, 0} \cap T\left(h_{\alpha, 1}+\rho_{\alpha, 0}\right)^{-1}\left(\frac{2}{3}, \infty\right)$ and $a_{\alpha, 0} \cap T\left(h_{\alpha, 1}+\rho_{\alpha, 0}\right)^{-1}\left(-\infty, \frac{1}{3}\right)$.

Let us note that for all $\rho \in C_{1}, T\left(\rho \cdot 1_{c_{\alpha, 0}}\right) \cdot 1_{a_{\alpha, 0}}$ converges to 0 . There is a countable set $\mathcal{E}_{\alpha, 1} \supset \mathcal{E}_{\alpha, 0}$ of predense subsets of $P_{h_{\alpha, 1}+\rho_{\alpha, 0}, d_{\alpha, 1} \cup a_{\alpha, 0}}$ with the property that so long as a filter $G$ with $h_{\alpha, 0}+\rho_{\alpha, 0} \in G$ meets each element of $\mathcal{E}_{\alpha, 1}$, it will ensure that $\operatorname{val}_{G}\left(\dot{Y}_{\beta_{0}}\right)$ does not split $a_{\alpha, 0} \cap T\left(h_{\alpha, 1}+\rho_{\alpha, 0}\right)^{-1}\left(\frac{2}{3}, \infty\right)$ and $a_{\alpha, 0} \cap T\left(h_{\alpha, 1}+\rho_{\alpha, 0}\right)^{-1}\left(-\infty, \frac{1}{3}\right)$.

We continue by choosing any $p_{1} \in P_{h_{\alpha, 1}+\rho_{\alpha, 0}, d_{\alpha, 1} \cup a_{\alpha, 0}}$ and any $\beta_{1}<\alpha$. We will select $\tilde{a}_{\alpha, 1}, c_{\alpha, 1}, a_{\alpha, 1}$ as subsets of $c_{\alpha, 0}$ as we did with $\tilde{a}_{\alpha, 0}, c_{\alpha, 0}, a_{\alpha, 0}$. We set $d_{\alpha, 2}=$ $\mathbb{N} \backslash\left(a_{\alpha, 1} \cup c_{\alpha, 1}\right)$ and $h_{\alpha, 2}$ as above so that $h_{\alpha, 2} \cdot 1_{d_{\alpha, 0} \cup a_{\alpha, 0}}=\left(h_{\alpha, 1}+\rho_{\alpha, 0}\right) \cdot 1_{d_{\alpha, 0} \cup a_{\alpha, 0}}$.

The recursion continues for $\omega$-many steps and we define $f_{\alpha+1}$ to be the unique function satisfying that $f_{\alpha+1} \cdot 1_{d_{\alpha} \cup a_{\alpha}}=f_{\alpha+1}$ and $f_{\alpha+1} \cdot 1_{d_{\alpha, \ell} \cup a_{\alpha, \ell}}=h_{\alpha, \ell}+\rho_{\alpha, \ell}$ for all $\ell \in \omega$. 
In this recursion, it is easily arranged that $\bigcup_{\ell}\left(d_{\alpha, \ell} \cup a_{\alpha, \ell}\right)=d_{\alpha} \cup a_{\alpha}=d_{\alpha+1}$ and let $\rho_{\alpha}=f_{\alpha+1} \cdot 1_{a_{\alpha}}$. Additionally, it is easily arranged that for each $n$ and each pair $p \in P_{h_{\alpha, n}, d_{\alpha, n}}, \beta<\alpha$, there is an $\ell \geq n$ such that at stage $\ell$ we are considering $p_{\ell}=p$ and $\beta_{\ell}=\beta$.

Choose any $q \in P_{f_{\alpha+1}, d_{\alpha+1}}$ and $\beta \in \alpha$. Choose any $k \in \omega$ so that the finite set of places where $q$ might disagree with $f_{\alpha+1}$ is contained in $d_{\alpha, k}$. Let $p=q\left\lceil d_{\alpha, k}\right.$ and choose $\ell>k$ so that at stage $\ell$ of this construction, we were considering $p$ and $\dot{Y}_{\beta}$. This means that at stage $\ell$, we were working with $q \uparrow d_{\alpha, \ell+1}$ and we arranged that $q \uparrow\left(d_{\alpha, \ell+1} \cup a_{\alpha, \ell}\right)$ forced over the poset $P_{h_{\alpha, \ell+1}+\rho_{\alpha, \ell}, d_{\alpha, \ell+1}}$ that $\dot{Y}_{\beta}$ did not mod finite split $a_{\alpha, \ell} \cap T\left(h_{\alpha, \ell+1}\right)^{-1}\left(\frac{2}{3}, \infty\right)$ and $a_{\alpha, \ell} \cap T\left(h_{\alpha, \ell+1}\right)^{-1}\left(-\infty, \frac{1}{3}\right)$. We set $\mathcal{E}_{\alpha, \ell+1}$ to be a countable family of predense sets that will ensure this continues to hold, and at stage $\ell+1$, we ensured that for all $\rho \in C_{1}$ such that $\rho \cdot 1_{a_{\alpha, \ell+1} \cup c_{\alpha, \ell+1}}=\rho$, each member of $\mathcal{E}_{\alpha, \ell+1}$ is predense in $P_{h_{\alpha, \ell+1}+\rho, \mathbb{N} \backslash c_{\alpha}}$. Define $\mathcal{D}_{\alpha+1}$ to be any countable collection of predense subsets of $P_{f_{\alpha+1}, d_{\alpha+1}}$ which contains $\mathcal{D}_{\alpha}$ and $\bigcup_{\ell} \mathcal{E}_{\alpha, \ell+1}$. Since $T\left(f_{\alpha+1}\right) \cdot 1_{a_{\alpha, \ell}}$ is asymptotically equal to $T\left(h_{\alpha, \ell+1}\right) \cdot 1_{a_{\alpha, \ell}}$, we have completed the proof of the lemma.

Lemma 6 Assume that $\left\{d_{n}: n \in \omega\right\}$ is an increasing family of members of $\mathcal{J}$ and that $\left\{h_{n}: n \in \omega\right\} \subset C_{1}$ has the property that, for each $n, h_{n+1} \cdot 1_{d_{n}}=h_{n}$. Then, for any countable family $\mathcal{E}$ of predense subsets of the poset $\bigcup_{n} P_{h_{n}, d_{n}}$, there is a pair $h \in C_{1}$ and $d \in \mathcal{J}$ such that $\bigcup_{n} P_{h_{n}, d_{n}} \subset P_{h, d}$ and each $E \in \mathcal{E}$ is predense in $P_{h, d}$.

Proof Similar to Lemma 3. First to choose $d$ we define $d \cap A_{n}$ for each $n$. Choose $d \cap A_{n}$ so that

(1) $A_{n} \cap d_{m} \subset d$ for each $m<n$,

(2) $\left(A_{n} \cap d_{m}\right) \backslash d$ is finite for all $m$,

(3) $A_{n} \backslash d$ is infinite.

Naturally we have ensured that $d \in \mathcal{J}$ and that $d_{m} \backslash d$ is contained in $\bigcup_{n \leq n} A_{n} \cap d_{m} \backslash d$, and so is finite. We will define $h$ so that $h \cdot 1_{d}=h$ and so that $h \cdot 1_{d_{n} \cap d}=h_{n} \cdot 1_{d_{n} \cap d}$ for each $n$. However, in order to ensure that each $E \in \mathcal{E}$ is still predense in $P_{h, d}$, we will recursively shrink $d$ while preserving that $d_{n} \backslash d$ is finite for all $n$. By recursion on $k$ we will choose a finite set $L_{k}$ disjoint from $d_{k}$, and will redefine $d$ to be $d \backslash \bigcup_{n} L_{n}$. Let $\left\{p_{k}, E_{k}: k \in \omega\right\}$ be an enumeration of all pairs from $\bigcup_{n} P_{h_{n}, d_{n}}$ and $\mathcal{E}$.

Suppose we have chosen $L_{k}$ and we consider the pair $p_{k}, E_{k}$. Choose $n_{k+1}$ large enough so that there is an $e \in E_{k}$ compatible with $p_{k}$ and so that both $e$ and $p_{k}$ are in $P_{h_{n}, d_{n}}$ for some $n<n_{k+1}$. In addition, assume that $\operatorname{dom}\left(p_{k}\right) \backslash d_{n}$ is contained in $\bigcup_{j<n_{k+1}} A_{j}$. 
Choose a finite set $L_{k+1} \subset d_{n_{k+1}} \backslash d_{k}$ so that $\left\{\ell:\left(e \cup p_{k}\right)(\ell) \neq h_{n_{k+1}}(\ell)\right\}$ is contained in $d_{k} \cup L_{k+1}$. It follows that we will have that $p_{k}$ and $e$ will be compatible in $P_{h, d}$.

\section{Acknowledgements}

The author acknowledges support provided by NSF grant DMS-0103985.

\section{References}

[1] Christina Brech and Piotr Koszmider, $\ell_{\infty}$-sums and the Banach space $\ell_{\infty} / c_{0}$, Fund. Math. 224 (2014), no. 2, 175-185; doi: 10.4064/fm224-2-3

[2] Alan Dow and Saharon Shelah, More on tie-points and homeomorphism in $\mathbb{N}^{*}$, Fund. Math. 203 (2009), no. 3, 191-210; doi: 10.4064/fm203-3-1

[3] Lech Drewnowski and James W. Roberts, On the primariness of the Banach space $l_{\infty} / C_{0}$, Proc. Amer. Math. Soc. 112 (1991), no. 4, 949-957; doi: 10.2307/2048638

[4] Magdalena Grzech, Set theoretical aspects of the Banach space $l_{\infty} / c_{0}$, Ann. Pure Appl. Logic 126 (2004), no. 1-3, 301-308, Provinces of logic determined; doi: 10.1016/j.apal.2003.11.023

[5] Ilijas Farah, Analytic quotients: theory of liftings for quotients over analytic ideals on the integers, Mem. Amer. Math. Soc. 148 (2000), no. 702, xvi+177; doi: 10.1090/memo/0702

[6] I. E. Leonard and J. H. M. Whitfield, A classical Banach space: $l_{\infty} / c_{0}$, Rocky Mountain J. Math. 13 (1983), no. 3, 531-539; doi: 10.1216/RMJ-1983-13-3-531

[7] A. A. Miljutin, Isomorphism of the spaces of continuous functions over compact sets of the cardinality of the continuum, Teor. Funkciu Funkcional. Anal. i Priložen. Vyp. 2 (1966), 150-156. (1 foldout).

[8] S. Negrepontis, The Stone space of the saturated Boolean algebras, Trans. Amer. Math. Soc. 141 (1969), 515-527; doi: 10.1090/S0002-9947-1969-0248057-2

[9] Elliott Pearl (ed.), Open problems in topology. II, Elsevier B. V., Amsterdam, 2007.

[10] Saharon Shelah, Proper forcing, Lecture Notes in Mathematics, vol. 940, SpringerVerlag, Berlin, 1982; doi: 10.1007/978-3-662-21543-2

[11] Saharon Shelah and Juris Steprāns, PFA implies all automorphisms are trivial, Proc. Amer. Math. Soc. 104 (1988), no. 4, 1220-1225; doi: 10.1090/S0002-9939-19880935111-X

[12] Juris Steprāns, The autohomeomorphism group of the Čech-Stone compactification of the integers, Trans. Amer. Math. Soc. 355 (2003), no. 10, 4223-4240 (electronic); doi: 10.1090/S0002-9947-03-03329-4 
[13] Stevo Todorčević, Analytic gaps, Fund. Math. 150 (1996), no. 1, 55-66.

[14] Boban Veličković, OCA and automorphisms of $\mathcal{P}(\omega)$ /fin, Topology Appl. 49 (1993), no. 1, 1-13; doi: 10.1016/0166-8641(93)90127-Y

Department of Mathematics, UNC-Charlotte, 9201 University City Blvd., Charlotte, NC 28223

adow@uncc. edu

http://math. uncc.edu/ adow

Received: 10 July 2015 Revised: 16 August 2016 